\title{
Notched UWB bandpass filter using complementary single split ring resonator
}

\author{
S. S. Karthikeyan ${ }^{\text {a) }}$ and Rakhesh Singh Kshetrimayum ${ }^{\text {b) }}$ \\ Department of Electronics and Communication Engineering, Indian Institute \\ of Technology Guwahati, Assam, India-781039 \\ a)ssk@iitg.ernet.in \\ b) krs@iitg.ernet.in
}

Abstract: In this paper, a simple method to obtain a notch in the ultra-wideband (UWB) bandpass filter is presented. UWB bandpass filter is designed using ground plane aperture technique. To reject the undesired wireless local area network (WLAN) signals, a complementary single split ring resonator (CSSRR) is placed under the coupled lines of the bandpass filter. The CSSRR has flat insertion loss and good roll off rate than the conventional complementary split ring resonator (CSRR). The proposed filter offers an ultra wide bandwidth bandpass filter characteristics with a steep notch function around 5.5 GHz. Hence the interference from the devices operated in the IEEE 802.11a spectrum can be avoided properly. IE3D simulation results of the notched UWB bandpass filter is in good agreement with the measurement results.

Keywords: complementary split ring resonator (CSRR), ultra wideband bandpass filter

Classification: Microwave and millimeter wave devices, circuits, and systems

\section{References}

[1] H. Shaman and J.-S. Hong, "Ultra-wideband (UWB) bandpass filter with embedded band notch structures," IEEE Microw. Wireless Compon. Lett., vol. 17, no. 3, pp. 193-195, March 2007.

[2] S. W. Wong and L. Zhu, "Implementation of compact ultra wideband bandpass filter with a notch-band," IEEE Microw. Wireless Compon. Lett., vol. 18, no. 1, pp. 10-12, Jan. 2008.

[3] G.-M. Yang, R. Jin, C. Vittoria, V. G. Harris, and N. X. Sun, "Small ultra-wideband (UWB) bandpass filter with notched band," IEEE Microw. Wireless Compon. Lett., vol. 18, no. 3, pp. 176-178, March 2008.

[4] A. Ali and Z. Hu, "Metamaterial resonator based wave propagation notch for ultra wideband filter applications," IEEE Antennas Wireless Propag. Lett., vol. 7, pp. 210-212, 2008.

[5] L. Qi, L. Chang-Hong, Z. Wei, and X. Wei-Feng, "Novel compact UWB bandpass filter with notched band," Microw. Opt. Tech. Lett., vol. 52, no. 2, pp. 280-283, Feb. 2010. 
[6] S. S. Karthikeyan and R. S. Kshetrimayum, "Slot split ring resonators and its applications in performance enhancement of microwave filter," IEEE Appl. Electromag. Conf., Kolkata, India, Dec. 2009.

[7] H.-W. Wu, M.-H. Weng, Y.-K. Su, C.-Y. Hung, and R.-Y. Yang, "Spurious suppression of a dualmode bandpass filter using simple c-shaped electromagnetic bandgap cell," Microw. Opt. Tech. Lett., vol. 48, no. 10, pp. 2090-2093, Oct. 2006.

[8] L. Zhu, H. Bu, and K. Wu, "Broadband and compact multi-pole microstrip bandpass filters using ground plane aperture technique," Microw. Opt. Tech. Lett., vol. 149, no. 1, pp. 71-77, 2002.

\section{Introduction}

The Federal Communication Commission (FCC) authorized the unlicensed use of UWB frequency spectrum (3.1-10.6 GHz) in 2002 for high data rate and short range communication. Since then, there has been a growing interest in the field of UWB communication. To fulfill the required UWB frequency range, various UWB filters have been developed in the past. But some of the frequency range $(5-6 \mathrm{GHz})$ within the UWB spectrum is allocated for wireless local area network (WLAN) systems. In order to avoid the interference between the UWB frequency spectrum and the WLAN radio signals, UWB bandpass filter with notch band is desirable. Till now various UWB $\mathrm{BPF}$ with notch band is reported by many researchers $[1,2,3,4,5]$. UWB bandpass filter with narrow band notch characteristic using embedded open stub is demonstrated in [1] and [5]. In [2], folded interdigital coupled lines are used to create rejection band at $5.6 \mathrm{GHz}$. Meander line slot etched in the active area of UWB BPF, effectively removed the IEEE $802.11 \mathrm{a}(5.15 \mathrm{GHz}$ to $5.85 \mathrm{GHz}$ ) signals from the UWB passband [3]. Recently, metamaterial resonator has been employed to introduce wave propagation notch in the UWB bandpass filter [4]. The filters mentioned above have the disadvantage of either low performance or difficult in fabrication due to via holes. In this work, we present an UWB bandpass filter with notch function using CSSRR. Compared to conventional CSRR, it gives a sharper cutoff transition and flat insertion loss after the resonance. Hence CSSRR is utilized to introduce a sharp rejection band in the WLAN frequency range. The IE3D predicted results of the proposed notched UWB filter are verified with the experimental results.

\section{Complementary single split ring resonator (CSSRR)}

Over the past few years, a new type of artificial materials called as metamaterials have attracted the attention of many researchers. Split ring resonator (SRR) and complementary split ring resonator (CSRR) are the most widely used planar structure for exhibiting metamaterial properties. Since CSRR is etched out from the ground plane, it occupies smaller area than the SRR operating at the same frequency. When excited by an axial time varying 
electric field, CSRR exhibits narrow stopband near its resonance frequency due to negative permittivity. The geometry of CSSRR and CSRR is shown in Fig. 1 (a) and Fig. 1 (b). Unlike conventional structure, CSSRR has only one split ring. This structure is designed on FR4 substrate with dielectric constant $\varepsilon_{r}=4.4 \mathrm{~mm}$ and height $\mathrm{h}=1.6 \mathrm{~mm}$ and placed under a $50 \Omega$ microstrip line. The dimensions of the CSSRR are as follows: $\mathrm{a}=11.45 \mathrm{~mm}, \mathrm{c}=0.5 \mathrm{~mm}$ and $\mathrm{d}=0.5 \mathrm{~mm}$. At resonance frequency of $2.04 \mathrm{GHz}$, the CSSRR exhibits sharp rejection band. It has been verified from a detailed parametric study, which has been reported elsewhere by the authors [6], that the approximate close-form formula for the resonance frequency of SSRR is given by:

$$
f_{r}=\frac{v}{2 \times\{4 \times(a-d)-c\} \sqrt{\frac{\varepsilon_{r}+1}{2}}}
$$

where $v$ is the speed of the light in vacuum. To validate the application of CSSRR for practical design, the equivalent lumped element model of CSSRR is shown in Fig. 1 (c). CSSRR is considered as parallel RLC resonant circuit. $\mathrm{L}$ and $\mathrm{C}$ are the inductance and capacitance of the CSRR and $R_{1}$ represents the losses in the resonant circuit. The nonuniform current distribution effects on the ground plane is modeled using $R_{2}$. The equivalent circuit parameters are easily obtained from the simulated scattering parameters with the help of the following equations [7],

$$
\begin{gathered}
A=\frac{\left(1+S_{11}\right)\left(1-S_{22}\right)+S_{12} S_{21}}{2 S_{21}}=1+\frac{Y_{1}}{Y_{2}} \\
B=Z_{0} \frac{\left(1+S_{11}\right)\left(1+S_{22}\right)+S_{12} S_{21}}{2 S_{21}}=\frac{1}{Y_{2}}
\end{gathered}
$$

where $Y_{1}$ and $Y_{2}$ are the parallel and series susceptance of the $\Pi$ - type symmetrical two port network. $Z_{0}$ is the characteristics impedance of the microstrip line. The relation between the equivalent circuit parameters and the $\Pi$ - type two port network are given by,

$$
Y_{1}=\frac{A-1}{B}=\frac{1}{R_{2}}+j B_{2} ; Y_{2}=\frac{1}{R_{1}}+j B_{1}
$$

since the simulated scattering parameters of the CSSRR are complex, the resulting equivalent circuit parameters are also complex, from which the lumped element values of CSSRR are obtained using Eq. (5).

$$
C=\frac{B_{1}}{2 \pi f_{0}\left(\frac{f_{0}}{f_{c}}-\frac{f_{c}}{f_{0}}\right)} ; L=\frac{1}{4 \pi^{2} f_{0}^{2} C}
$$

where $f_{0}$ and $f_{c}$ are attenuation pole frequency and cutoff frequency of the CSSRR respectively. The extracted lumped element values of the structure are $L=1.6624 \mathrm{nH}, C=3.665 \mathrm{pF}, R_{1}=0.833 \mathrm{~K} \Omega$ and $R_{2}=1.1 \mathrm{~K} \Omega$ at the resonant frequency $2.04 \mathrm{GHz}$. The equivalent circuit of CSSRR is simulated using Ansoft Designer SV and compared with the electromagnetic simulation. The result is shown in Fig. 1(d). EM simulated results are well matched with the circuit simulation. Small differences may be due to the negligence of fringe 


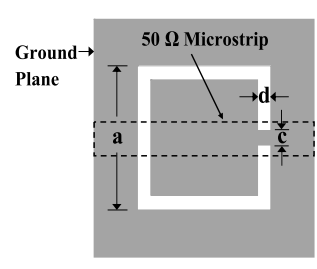

(a)

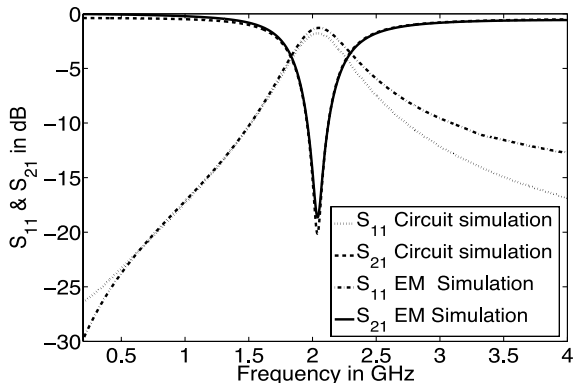

(d)

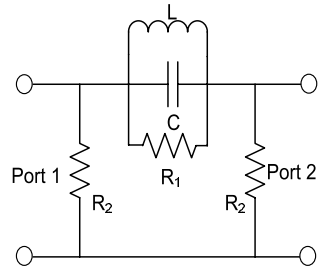

(c)

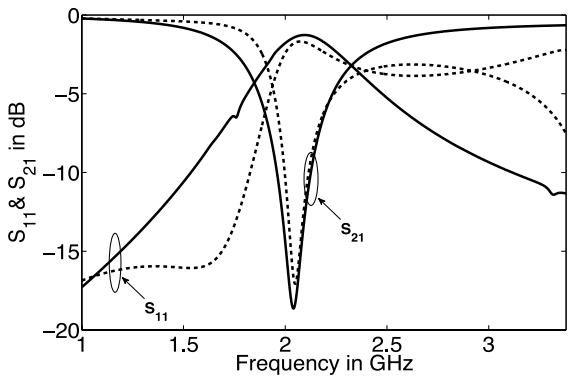

(e)

Fig. 1. Geometry of a) Proposed CSSRR b) Conventional $\operatorname{CSRR}(\mathrm{a}=10 \mathrm{~mm}, \mathrm{~d}=\mathrm{g}=\mathrm{c}=0.5 \mathrm{~mm}) \mathrm{c}$ ) Lumped element model of CSSRR d) Scattering parameters of CSSRR e) Simulated scattering parameters of CSRR and CSSRR (CSRR - Dashed line, CSSRR - Solid line)

field capacitance in the model. Conventional CSRR for the same resonance frequency of CSSRR is simulated for the comparison purpose. Fig. 1 (e) illustrates the frequency response of CSRR and CSSRR. By observing the plots, we can conclude that conventional CSRR insertion loss is high and is not having flat response after resonance. Similarly the roll-off rate of return loss is also low after the resonant frequency. So the conventional CSRR performance is poor for notch function. In order to improve the performance of the conventional CSRR, it was slightly etched away from the host line and an open circuited stub was employed to compensate the increase in inductance [4]. But by using CSSRR, above the resonant frequency, we can achieve flat insertion loss in the passband and better roll-off rate without adding any additional structure.

\section{Bandpass filter with notched band}

To design the UWB bandpass filter with notch function, UWB BPF is designed first using ground plane aperture technique [8]. Due to the aperture in the ground plane, close coupling between the microstrip lines are achieved. Layout and fabricated prototype of UWB BPF are shown in Fig. 2 (a) and 2 (b). A microstrip multiple mode resonator (MMR) line is used to link the two identical parallel coupled microstrip line (PCML) sections with a backside aperture. The detailed design procedure of UWB BPF can be obtained from [8]. The width and the gap between the coupled lines of a parallel coupled microstrip line (PCML) are $0.2 \mathrm{~mm}$. The length of the PCML section is 


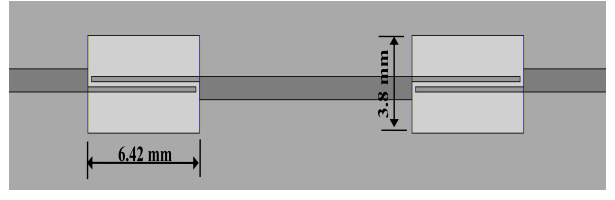

(a)

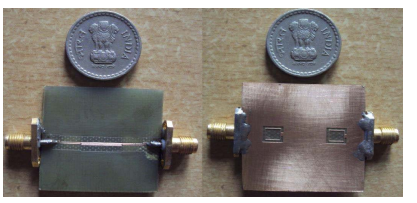

(b)

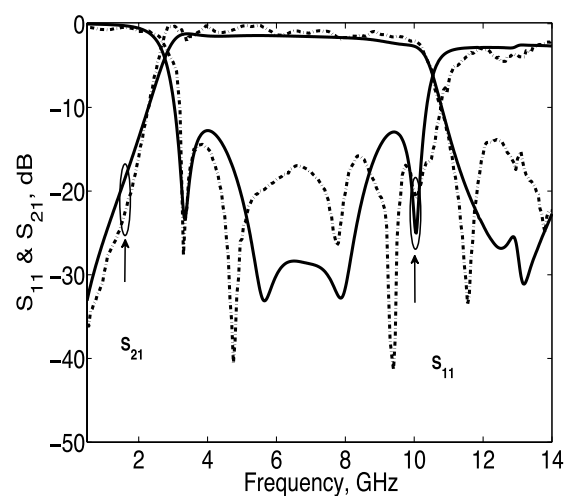

(c)

Fig. 2. UWB BPF with aperture in the ground plane a) Geometry b) Photograph c) Simulated (solid line) and measured (dashed line) scattering parameters

$6.32 \mathrm{~mm}$ which is equivalent to the electrical length $\phi=180^{\circ}$. The physical length of a multi mode resonator is equal to $12.25 \mathrm{~mm}$. The length of the ground plane aperture is $6.42 \mathrm{~mm}$ and the height is $3.8 \mathrm{~mm}$, which are optimized using EM simulation. Fig. 2 (c) shows the simulated and measured scattering parameters of UWB BPF. The filter passband is from $2.8 \mathrm{GHz}$ to $10.6 \mathrm{GHz}$. The measured insertion loss of conventional UWB BPF is less than $1.5 \mathrm{~dB}$ in the passband. Better results can be obtained using low loss substrate. As discussed earlier, to notch out the WLAN radio signals from the desired UWB passband, stopband characteristics of a CSSRR is utilized. The CSSRR resonating at $5.5 \mathrm{GHz}$ is designed and is placed underneath the parallel coupled lines. The initial dimensions of CSSRR is obtained from (1). Since the CSSRR is etched around the ground plane aperture, resonant frequency of the CSSRR is not exactly matched with the equation (1). However the final dimensions are optimized using EM simulation. To provide the room for both the aperture and the CSSRR under the coupled lines, size of the aperture is modified. Similarly, instead of square shape CSSRR, rectangular shape CSSRR is used to obtain the proper notch out behavior. All the specifications of UWB BPF with notched band and photograph of the fabricated filter are shown in Fig. 3 (a) and Fig. 3(b). Fig. 3(c) shows the simulated and experimental results of UWB BPF. The fabricated UWB BPF passband is from $2.96 \mathrm{GHz}$ to $10.75 \mathrm{GHz}$ exhibiting notch characteristics at $5.5 \mathrm{GHz}$ with a rejection level of $32.25 \mathrm{~dB}$. The lower and upper $3 \mathrm{~dB}$ points of notch are $4.85 \mathrm{GHz}$ and $6.12 \mathrm{GHz}$ respectively. So the proposed filter is capable of suppressing the interference from IEEE 802.11a WLAN signals effectively. Compared to the work reported in [1] and [2], the proposed method 


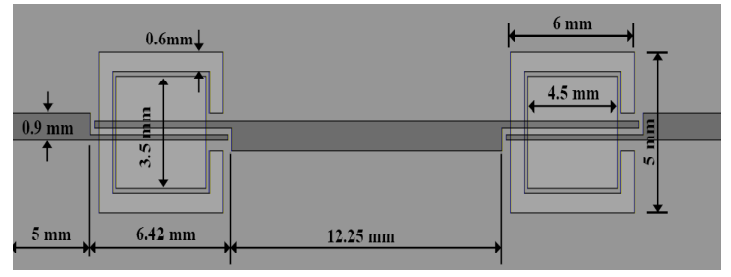

(a)

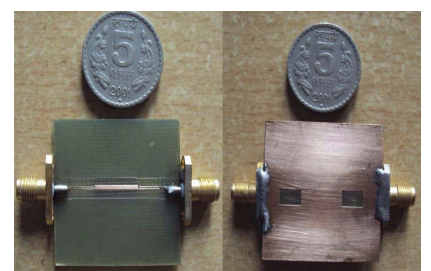

(b)

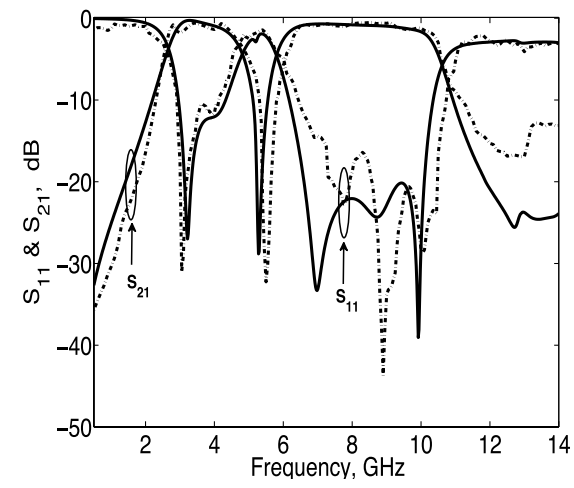

(c)

Fig. 3. Proposed UWB BPF with notched band a) Geometry b) Photograph c) Simulated (solid line) and measured (dashed line) scattering parameters

provides better $10 \mathrm{~dB}$ FBW in the notch band and does not require any via holes as used in [1] and [5].

\section{Conclusion}

In this work, CSSRR is suggested instead of CSRR for obtaining good stopband performance. Better rejection characteristic is obtained without additional design guidelines such as addition of open stubs and placing of CSRR away from the host line. By placing the CSSRR under the coupled lines of UWB BPF, notch response is obtained in the passband of BPF. Good agreement was obtained between the simulated and the fabricated filters. The fabricated filter has UWB bandpass response with FBW of $113 \%$ at the midband frequency of $6.85 \mathrm{GHz}$ and a narrow notch in the passband with $10 \mathrm{~dB}$ FBW, which is about $9.09 \%$ at the center frequency of $5.5 \mathrm{GHz}$. Maximum rejection level in the notch band is $32 \mathrm{~dB}$. So, the proposed UWB BPF with notch function will effectively pass the UWB signals without any interference from the WLAN radio signals.

\section{Acknowledgements}

Authors are grateful to the Science and Engineering Research Council, Department of Science and Technology, Government of India for supporting this study (Project No: ECE/P/KRS/DST/01). 